\title{
Late-onset congenital syphilis: is it another disease?
}

\author{
Sífilis congênita tardia: trata-se de outra doença?
}

Won Sriwijitalai', Viroj Wiwanitkit ${ }^{2}$

Dear Editor,

We read the publication on "Late-onset congenital syphilis with unusual brain abnormalities" with great interest ${ }^{1}$. The atypical neurological finding and interesting blood and cerebrospinal fluid (CSF) serological findings are reported and Silva et al. ${ }^{1}$ concluded that this case was a late-onset congenital syphilis. This may or may not be a late-onset congenital syphilis. The main points that might not support the diagnosis of late-onset congenital syphilis are: a) the lack of additional clinical history of other clinical features of late syphilis, b) the negative CSF VDRL, c) no clinical data on the present syphilis status of the patient's mother and d) the uncommon neurological imaging finding. False positive and negative results may have occurred in the present case ${ }^{2}$. The clinical finding and laboratory result may be features of other diseases, especially autoimmune diseases ${ }^{3}$. The lack of a CSF PCR test for final confirmation of neurosyphilis infection results in an inconclusive diagnosis. Nevertheless, in case that it is a real late syphilis with an atypical neurological feature, the authors cannot make a diagnosis that this is a congenital case with confirmed vertical transmission since is not possible to rule out the possibility of a non-vertical transmission in this female adolescent.

\section{References}

1. Silva RAE, Campelo C, Godeiro-Junior C.

Late-onset congenital syphilis with

unusual brain abnormalities. Arq

Neuropsiquiatr. 2017 Sep;75(9):676.

https://doi.org/10.1590/0004-282×20170118
2. Nishii H, Matsumoto T. [Tests for syphilis]. Nihon Rinsho. 2005 Jul;63 Suppl 7:140-1. Japanese.

3. Nandwani R, Evans DT. Are you sure it's syphilis? A review of false positive serology. Int J STD AIDS. 1995 JuL-Aug;6(4):241-8. https://doi.org/10.1177/095646249500600404 